\title{
GENERAL
}

\section{COMPARISON OF CANCER PATIENTS' QUALITY OF LIFE IN HUNGARY AND GERMANY - A CROSS-NATIONAL QUESTIONNAIRE ANALYSIS}

\author{
JOHANNES THOMA, ROMÁNA ZELKÓ* and BALÁZS HANKÓ
}

Faculty of Pharmacy, University Pharmacy Department of Pharmacy Administration, Semmelweis University, Hogyes E. St. 7-9, H-1092 Budapest, Hungary

\begin{abstract}
Approaches investigating cancer patients' quality of life have been conducted intensely but most approaches either focusing on the quality of life analysis of patients living in the same country or suffering from the same cancer type. The aim of this study was to compare cancer patients' quality of life in Hungary and Germany. A self - developed questionnaire was distributed in 26 community pharmacies and 4 hospitals in Hungary and Germany from August 2013 to October 2014. Pharmacy selection was performed by reflecting on proper questionnaire randomization. Based on the available facilities the objective was to give a preferably diversified picture of cancer patients' QoL in the two countries. As the quality of life is a subjective parameter, the patients were asked to make a cross on a scale of 1 to 100 . Patients were screened by hospital and pharmacy staff by application of inclusion and exclusion criteria. The non-parametric Mann-Whitney and Wilcoxon W-test methods were used to analyze differences in the mean of estimated quality of life between Hungary and Germany. A difference $(\mathrm{p}=0.043)$ in the quality of life mean between Hungarian $(65.92 \pm 19.25)$ and German $(57.35 \pm 23.45)$ cancer patients was observed. The evaluated median of estimated quality of life differed in Hungary (70) and Germany (60). The interquartile ranges of both compared groups were 30. The result is not in accordance with the general quality of life index of the whole population in Hungary and Germany. There seem to be determining factors which influence the quality of life assessment of cancer patients, such as social relationships, the relation to individual standards and varying illness perceptions in different countries.
\end{abstract}

Keywords: cancer, quality of life, questionnaire, Hungary, Germany

Cancer has become a globally snowballing problem. It is the second leading cause of death and is expected to get worse simply due to the growth and ageing of the population (1-2). The diagnosis of cancer often means there is little time left for the affected patients. Therefore, besides scientific progress and promising therapeutic approaches, one has to bring more attention to the quality of life (QoL) parameter. The aim of the present study was to compare cancer patients' QoL in Hungary and Germany. Approaches investigating cancer patients' QoL have been conducted intensely but most approaches either focusing on the QoL analysis of patients living in the same country or suffering from the same cancer type. Since there is no other crossnational approach comparing cancer patients' QoL in Hungary and Germany, this approach can be considered as novel in this modality.

\section{EXPERIMENTAL}

A self-developed questionnaire was distributed in 26 community pharmacies and 4 hospitals in Hungary and Germany from August 2013 to October 2014. Pharmacy selection was performed by reflecting on proper questionnaire randomization. Based on the available facilities the objective was to give a preferably diversified picture of cancer patients' QoL in the two countries. Therefore one primarily performed classification of the questionnaire distribution places into hospitals and community pharmacies. Then the community pharmacies were classified in town- (inhabitants $>20,000$ ) and village pharmacies (inhabitants $<20,000$ ). Additionally one tried, on the one hand, to manage the distribution in a way that approximately equal numbers of valid questionnaires could be received in

\footnotetext{
* Corresponding author: e-mail: zelko.romana@pharma.semmelweis-univ.hu
} 
community pharmacies and hospitals. On the other hand, one intended to receive approximately equal numbers of questionnaires in town and village pharmacies. Therefore it was necessary to assume the patient flow characteristic. First of all seasonal fluctuation has to be taken in concern. The questionnaire distribution persisted several months. In many pharmacies, the patient flow in December is up to one third higher compared to the month of August. Additionally, accurate numbers of patient flow are difficult to receive, apply to the company secret and only a few pharmacy owners would be willing to relinquish these sensitive data. Therefore one had to assume the patient flow characteristic according to rational consideration. In both countries, there is a general trend that young people move to major towns and their parents and grandparents remain in the villages accompanying a lower average age in towns. Major towns offer a magnitude of important factors for young people. Just to name a few there may be universities allowing academic studies or broader possibilities to enjoy the off time. Accordingly in towns there often is a higher amount of active working population and consecutively superior spending power. Against this background, the expectation was to deal in town pharmacies with increased patient flow and consecutively with increased rates of questionnaire return compared to village pharmacies. Hence approximately triply village pharmacies were provided with questionnaires compared to the number of town pharmacies. 234 questionnaires were distributed in Germany and 180 in Hungary, making a total of 414. Finally, 73 questionnaires were received in hospitals, 40 questionnaires in town pharmacies and 35 questionnaires in village pharmacies, making a total of 148 . There was a statistical calculation before questionnaire distribution referring to the required number of questionnaires with the target of receiving valid and representative results. Presuming a confidence interval of $95 \%$ and a maximal tolerable sample error of $10 \%$, the intention was to receive at least 100 questionnaires for evaluation. It was calculated with a basic population of $1,000,000$ patients. Due to the sensitive patient pool $25 \%$ rate of response was calculated; hence 400 questionnaires were intended for distribution. To avoid bias and to achieve proper randomization, the distribution points of questionnaires were elected in all cardinal directions in the surroundings of Budapest in Hungary and Regensburg in Germany and deliberately excluded pharmacies within a radius of $5 \mathrm{~km}$ to oncologists. If the pharmacy or hospital agreed we left $10-15$ questionnaires at each distribution place. The proce- dure was continued up to the distribution of 400 questionnaires. Patients were screened by hospital and pharmacy staff by application of inclusion and exclusion criteria and were informed about the aim of the study. Pharmacy and hospital staff was not allowed to help the patients if any question came up. The questionnaire was anonymous and participants could decide if they wanted to answer directly in the pharmacy, hospital or at home. A self-developed questionnaire was used instead of a common tool or the often used EORTC - QLQ - 30 because those questions were not specific enough for the points elected to explore. Besides the QoL, one attached importance to the analysis of parameters which could have influenced the assessment of cancer patients' QoL. Just to name an example, the analysis of the stage of the disease was important which is not requested in the common tools. Since the questionnaire is not based on any of those common tools, the questionnaire was proofed for suitability by asking five physicians to check it. Thereby, inappropriate questions or ambiguity were avoided and validity and comprehensibility were maintained. To ensure reasonability and acceptance, the length of our questionnaire with 23 questions was adapted to the length of the EORTC - QLQ - C30. Due to the sensitive patient pool, there was no test run with a similar patient group.

The distributed questionnaire contained 23 questions and was the basis for the present study and the recently published study discussing the use of community pharmacists in cancer care (3). For the present study, question number 22.1 was evaluated to compare cancer patients' QoL in Hungary and Germany. As QoL is a subjective parameter, patients were asked to make a cross on a scale of 1 to 100 . To evaluate parameters which could have influenced the QoL assessment, the questions numbers $4,5,12$ and 19.2 were inquired to evaluate differences in cancer types, differences in the stage of the disease and disparities in the pain level or side effects experienced. The whole questionnaire and the datasets generated or analyzed during the current study are available from the corresponding author on reasonable request.

\section{Inclusion criteria}

Patients visiting the elected pharmacies were requested to answer the questionnaire if they submitted a prescription with an anticancer drug mentioned in a defined list. Hospitalized patients who were treated with such a drug were included as well. The list was developed by the investigator JT and checked by the two other investigators HB and RZ. 
The list included all prescription drugs in the German market containing an oncology indication up to the time of distribution in August 2013. The list can be apprehended upon request.

\section{Exclusion criteria}

If patients were not able to answer the questionnaire due to physical or psychical restrictions, they were excluded from the study. Patients who were reluctant to answer were also excluded.

\section{Statistical analysis}

The evaluation was performed by descriptive analysis. Due to the sample size, the non-parametric Mann-Whitney and Wilcoxon W-test methods were used to analyze differences in the mean of estimated QoL between Hungary and Germany. The one-sample Kolmogorov-Smirnov test method was used to identify the kind of distribution in both groups. For the analysis of parameters which could have influenced the QoL assessment such as the stage of the

Table 1. Estimated level of cancer patients' QoL in Hungary.

\begin{tabular}{|c|c|c|c|c|c|c|}
\hline & & \multirow{3}{*}{ Statistic } & \multicolumn{4}{|c|}{ Bootstrap $^{b}$} \\
\hline & & & \multirow{2}{*}{ Bias } & \multirow{2}{*}{ Std. Error } & \multicolumn{2}{|c|}{$95 \%$ Confidence interval } \\
\hline & & & & & Lower & Upper \\
\hline \multirow{2}{*}{$\mathrm{N}$} & Valid & 49 & & 6 & 38 & 60 \\
\hline & Missing & 0 & 0 & 0 & 0 & 0 \\
\hline \multicolumn{2}{|c|}{ Mean } & 65.9184 & -0.0215 & 2.7222 & 60.0000 & 71.0701 \\
\hline \multicolumn{2}{|c|}{ Median } & 70.0000 & -0.2600 & 3.0971 & 60.0000 & 80.0000 \\
\hline \multicolumn{2}{|c|}{ Mode } & $70.00^{\mathrm{a}}$ & & & & \\
\hline \multicolumn{2}{|c|}{ Std. deviation } & 19.24820 & -0.18589 & 1.98193 & 15.24921 & 23.06492 \\
\hline \multirow{3}{*}{ Percentiles } & 25 & 50.0000 & 1.6150 & 4.4468 & 45.0000 & 60.0000 \\
\hline & 50 & 70.0000 & -0.2600 & 3.0971 & 60.0000 & 80.0000 \\
\hline & 75 & 80.0000 & -0.2450 & 2.0449 & 70.0000 & 80.0000 \\
\hline
\end{tabular}

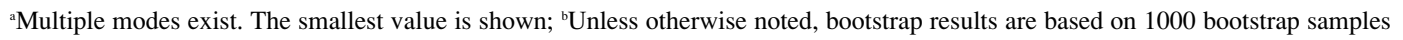

Table 1.1. Estimated level of cancer patients' QoL in Hungary - raw data.

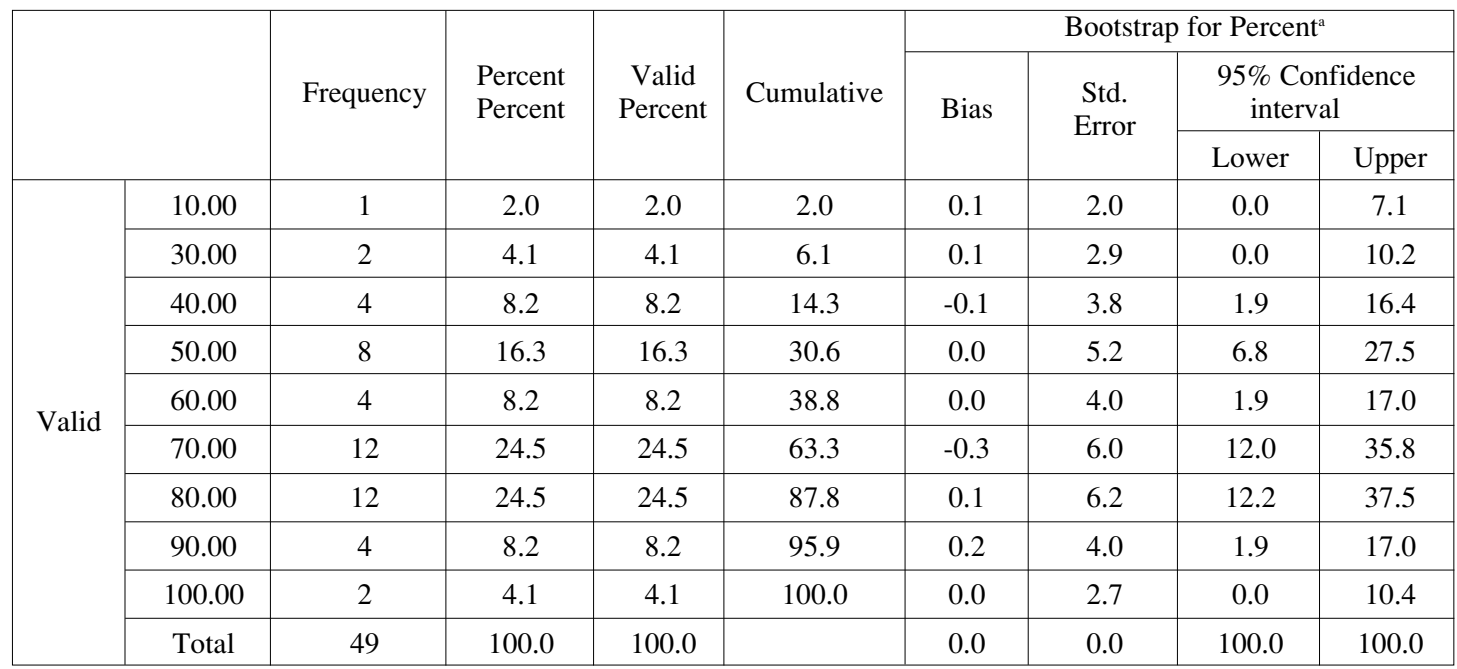

${ }^{a}$ Unless otherwise noted, bootstrap results are based on 1000 bootstrap samples 
disease, experiences with side effects or the type of cancer, the Fisher exact test method was used to evaluate differences between Hungary and Germany. The Mann-Whitney test was used for the evaluation of differences in the pain level of Hungarian and German cancer patients.

\section{Limitations}

Executing staff was asked to address the patients and to document successful approaches and failed approaches as well. Since the executing staff in pharmacies and hospitals worked only partly in accordance with this guideline, the evaluation of the

Table 2. Estimated level of cancer patients' QoL in Germany

\begin{tabular}{|c|c|c|c|c|c|c|}
\hline & & \multirow{3}{*}{ Statistic } & \multicolumn{4}{|c|}{ Bootstrap $^{b}$} \\
\hline & & & \multirow{2}{*}{ Bias } & \multirow{2}{*}{ Std. Error } & \multicolumn{2}{|c|}{$95 \%$ Confidence interval } \\
\hline & & & & & Lower & Upper \\
\hline \multirow{2}{*}{$\mathrm{N}$} & Valid & 79 & & 6 & 68 & 89 \\
\hline & Missing & 0 & 0 & 0 & 0 & 0 \\
\hline \multicolumn{2}{|c|}{ Mean } & 57.3544 & 0.1480 & 2.6455 & 52.1291 & 62.5000 \\
\hline \multicolumn{2}{|c|}{ Median } & 60.0000 & 1.2800 & 4.3509 & 50.0000 & 70.0000 \\
\hline \multicolumn{2}{|c|}{ Mode } & $70.00^{\mathrm{a}}$ & & & & \\
\hline \multicolumn{2}{|c|}{ Std. deviation } & 23.44609 & -0.34246 & 1.48849 & 20.14988 & 25.97786 \\
\hline \multirow{3}{*}{ Percentiles } & 25 & 40.0000 & 0.0400 & 7.6080 & 30.0000 & 50.0000 \\
\hline & 50 & 60.0000 & 1.2800 & 4.3509 & 50.0000 & 70.0000 \\
\hline & 75 & 70.0000 & 3.5625 & 4.6770 & 70.0000 & 80.0000 \\
\hline
\end{tabular}

${ }^{b}$ Unless otherwise noted, bootstrap results are based on 1000 bootstrap samples

Table 2.1. Estimated level of cancer patients' QoL in Germany - raw data.

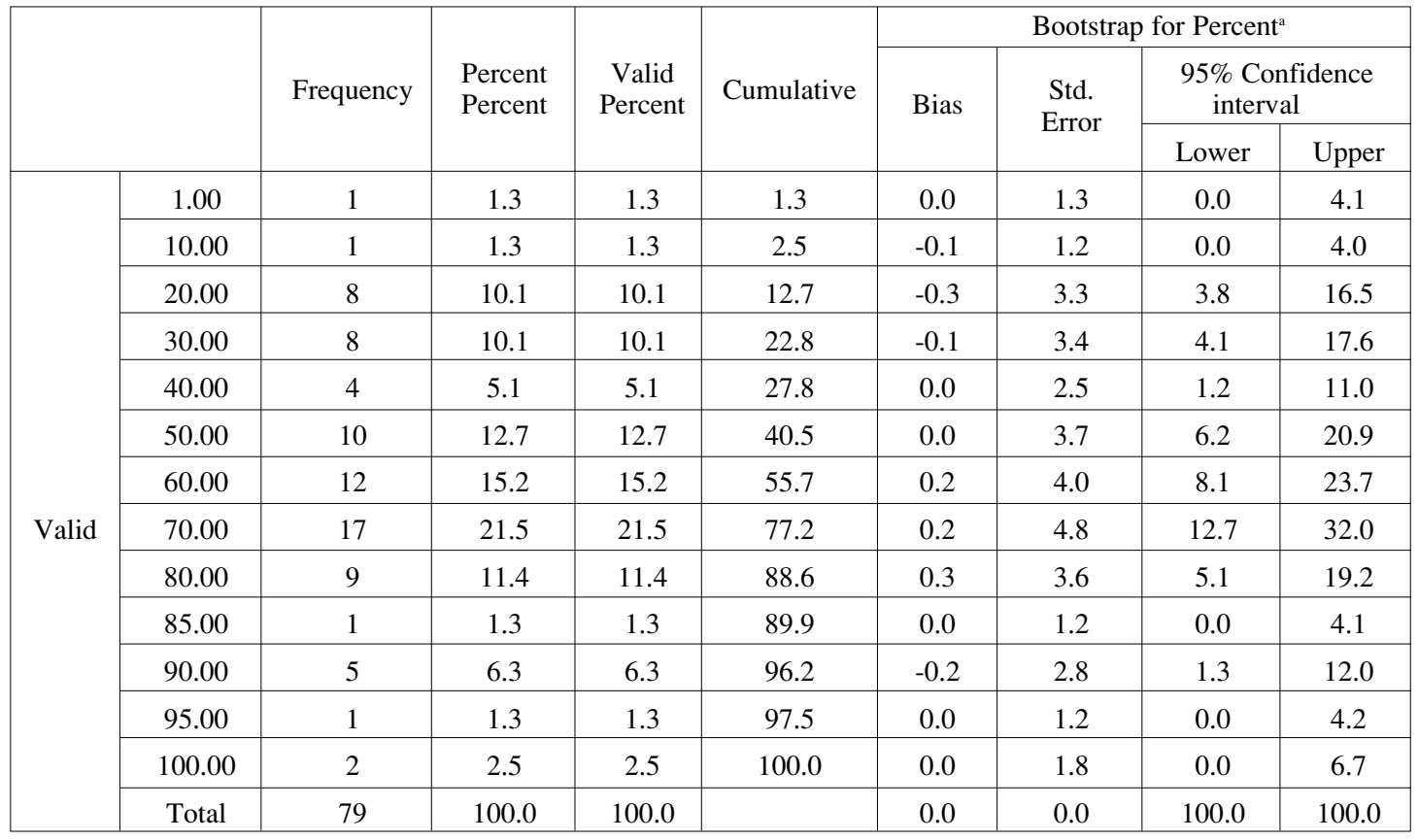

${ }^{a}$ Unless otherwise noted, bootstrap results are based on 1000 bootstrap samples 
rate of response was not possible. Additionally, one cannot exclude the possibility that help was received from family members when patients decided to answer the questionnaire at home. Due to the dependence on the voluntary benevolence of the pharmacy owners and even more important on the collaboration with pharmaceutical staff in community pharmacies and hospitals this study is limited to the number of selected pharmacies and hospitals. The number of questionnaires is limited to a sensitive patient pool. In many cases, such patients are busy with other thoughts than responding to a questionnaire.

The result of this study is limited to the additional use of drugs which could have influenced the evaluated QoL results. In future studies, it will be important to create groups of anticancer drugs and other drugs used for the treatment of the subject to analyze their impact on QoL. Precondition for the classification of drugs used for the treatment is the existence of the complete medication. This questionnaire was not construed for the analysis of the complete concurrent medication. All probands were required to answer the questionnaire separately and to receive no help from qualified staff or family members. Due to their physical condition and due to their level of information a multitude of the probands wouldn't have been able to note down their full medication in a proper way. The electronic health card will be the next step in the German health system to consolidate the whole current patient medication received by physician prescription and at the recommendation of pharmacies. Thereby it could be easier for future studies to identify the current medication and then to classify the drugs. Then the investigation of the QoL of patients who did not receive anticancer drug treatment and patients who received anticancer treatment could be performed, too. The current study does not contain this QoL comparison.

Furthermore, the study is limited to the type of cancer itself which can affect the QoL of the subjects. There were two main cancer types evaluable in both countries. In Germany breast cancer (46.5\%) was the most common one. In Hungary, bowel cancer occurred most frequently (51.6\%). The mentioned two groups represent a superior number in the particular countries and the remaining subjects split into a magnitude of diverse cancer types, which leads to inadequate numbers of subjects when evaluating cancer patients QoL referring to all single cancer types. The dependence on QoL and cancer type should be approached as one of the next steps in the future investigation of cancer patients QoL.
Therefore a wider and more extensive questionnaire distribution is necessary.

\section{RESULTS}

On a scale of 1 to 100 , a difference $(\mathrm{p}=0.043)$ was observed in the estimated QoL of Hungarian $(65.92 \pm 19.25)$ and German $(57.35 \pm 23.45)$ cancer patients. The evaluated median of estimated QoL also differed in Hungary (70) and Germany (60). German data distribution was normally $(\mathrm{p}=0.62)$. Hungarian data were not normally distributed $(\mathrm{p}=$ 0.046). The interquartile ranges (IQR) of both compared groups were 30 . Above mentioned results are illustrated in Tables 1 and 2. Raw data are illustrated in Tables 1.1 and 2.1. The evaluation of parameters which could have influenced the QoL assessment showed no differences in the stage of the disease $(\mathrm{p}=0.286)$ and side effects experienced $(\mathrm{p}=$ 0.821 ) by cancer patients in both countries. But there was a difference $(\mathrm{p}=0.005)$ regarding assessed pain level on a scale of 1 to 10 between Hungarian (6.67 \pm 0.39$)$ and German (5.21 \pm 0.33$)$ cancer patients. Cancer types also differed in both groups $(\mathrm{p}<0.001)$. In Germany, there was an excess of breast cancer (46.5\%). In Hungary, bowel cancer (51.6\%) was observed as the most frequent type. Raw data can be apprehended upon request.

\section{DISCUSSION}

Comparing the general QoL index of the whole population in Hungary and Germany in the year of questionnaire distribution, in the European ranking Hungary is ranked in place 23 and Germany in place 2 (4). The results of this study analyzing cancer patients' QoL are not in agreement with this data. In contrast, the QoL of cancer patients in Hungary was assessed to be higher than that of Germany. This outcome indicates a difference between general QoL and cancer patients' QoL, which hypothesizes the influence of other factors on cancer patients QoL. There was an attempt to evaluate which factors could have influenced this result. The stage of the disease did not occur as a determining factor less than experiences with side effects because in these points there was no difference between Hungary and Germany. Since the pain level of Hungarian cancer patients was assessed higher compared to German patients, the factor pain cannot be considered as a determining factor for the analyzed difference in the estimated QoL, too. This correlates with literature data in that the factor pain does not have a major impact on cancer patients' QoL (5). Also, the factor 
cancer type cannot be considered as determining for the analyzed difference in the estimated QoL. Giving the results of the current study there is an increased occurrence of bowel cancer in Hungary but literature data prove the direct and indirect influence of bowel cancer symptoms on QoL. Diarrhea, incontinence, and fatigue, for example, restrict bowel cancer patients' daily activities and their social life (6). Considering further factors the parameter age may not be disregarded. The mean age of cancer patients in Hungary was low compared to Germany. Especially the percentage of German patients older than 75 is conspicuous. But it is difficult to discuss the parameter age because it is a matter of opinion when interpreting the influence of the parameter age on the QoL of cancer subjects. On one hand, the less mean age in Hungary could have caused a less number of Hungarian subjects suffering from diverse age-related additional diseases. This could be an explanation for the elevated QoL of Hungarian patients compared to German subjects. On the other hand, the short mean age in Hungary is an indication that people fall ill in younger years. This allows the presumption that the QoL drops down to an earlier time compared to German subjects. Furthermore because diseases increase in the same way as the life age one could believe that patients falling ill high aged exhibit a generally reduced QoL. All aspects should be considered when interpreting the parameter age. Furthermore one has to take the statement of the WHO into account that cancer patients' perception of QoL depends not only on disease- and treatmentrelated factors but also on social relationships and individual value systems in relation to individual expectations and standards (7-8). In this context, the current results are plausible considering that patients hallmarked by a comparably lower QoL before cancer diagnosis have modest and adjusted expectations from their future life compared with patients hallmarked by a higher QoL in a healthy state accompanying exalted expectations from the future. This could subsequently lead to a subjective short QoL assessment of the high QoL patients after a cancer diagnosis. Patients' cultural contexts also have to be considered when targeting cancer patients' QoL (9). Furthermore, Fischer et al. recently analyzed differences in illness perceptions between two countries which also appear to influence QoL (10). The abovementioned points show the present result to be plausible and illustrate the complex issue when discussing the comparison of cancer patients QoL in the two countries. Finally, psychosocial factors such as integration and social support highly affect cancer patients' QoL $(9,11)$. The rising burden of cancer patients suffering from diminished QoL and the shortage of resources offering social support pose a difficult context. The pharmaceutical relevance was already discussed by performing a broad literature review about existing models of pharmaceutical interventions in oncology care. Pharmacists' inclusion in pain and symptom control teams, as well as home visitations helping patients with a conversation about their daily needs and problems, constitute only two of the services that could lift cancer patients' QoL to a higher level (12). There also was an investigation inquiring patients' expectations from pharmacists to raise up their QoL conditions. The most important point for patients was to have persons they can talk to medical and mainly from the human perspective. In the view of cancer patients, community pharmacists' skills were assessed most capable of completing this demand - qualified and with an adequate time schedule (3).

Compared to hospitals there is the possibility of a fast and continuous contact with community pharmacists which often generates a tight and personal relationship between a cancer patient and community pharmacist. This seems to be of great importance for cancer patients in their everyday life and affects their QoL in a positive way.

\section{CONCLUSION}

The QoL assessment of cancer patients in Hungary in Germany is not in accordance with the general QoL index of the whole population in Hungary and Germany. There seem to be determining factors which influence the quality of life assessment of cancer patients, such as social relationships, the relation to individual standards and varying illness perceptions in different countries.

\section{Acknowledgment}

The authors thank Andrea Meskó from Semmelweis University Budapest for providing statistical analysis for this study.

The manuscript does not include any individually identifiable health information. Patients asked voluntarily and were informed about the aim of the study accordingly. Therefore ethical approval was not required. All authors have nothing to disclose. There were no competing interests and no sponsor or funding sources, which supported this study. 


\section{REFERENCES}

1. http://www.who.int/mediacentre/factsheets/ fs297/en/ (accessed 15. 02. 2017).

2. https://www.cancer.org/research/cancer-facts statistics/global.html (accessed 15 February 2017).

3. Thoma J., Zelkó R., Hankó B.: Acta Pol. Pharm. 75, 229 (2018).

4. https://www.numbeo.com/quality-of-life/rankings by country.jsp?title=2017-mid\&region= 150 (accessed 09. 08. 2017).

5. Arndt V., Stegmaier C., Ziegler H., Brenner H.: Cancer 107, 2496 (2006).

6. Marventano S., Forjaz M.J., Grosso G., Mistretta A., Giorgianni G., Platania A.: BMC Surg. 132, 13 (2013).
7. http://www.who.int/healthinfo/survey/whoqolqualityoflife/en/ (accessed 05. 08. 2017).

8. Singer S., Langendijk J., Yarom N.: Am. Soc. Clin. Oncol. Educ. Book (2013).

9. Shim E.J., Mehnert A., Koyama A., Cho S.J., Inui H. et al.: Breast. Cancer. Res Treat. 99, 341 (2006).

10. Fischer M.J., Inoue K., Matsuda A., Kroep J.R., Nagai S. et al.: Breast. Cancer. Res. Treat. (2017).

11. Leung J., Pachana N.A., McLaughlin D.: Psychooncology 23, 1014 (2014).

12. Thoma J., Zelkó R., Hankó B.: Int. J. Clin. Pharm. 38, 855 (2016).

Received: 05.01.2018 